\title{
Functional dose-volume histograms for predicting radiation pneumonitis in locally advanced non-small cell lung cancer treated with late-course accelerated hyperfractionated radiotherapy
}

\author{
DONGQING WANG ${ }^{1,2}$, BAOSHENG $\mathrm{LI}^{1}$, ZHONGTANG WANG ${ }^{1}$, \\ JIAN ZHU ${ }^{3}$, HONGFU SUN ${ }^{1}$, JIAN ZHANG ${ }^{1}$ and YONG YIN ${ }^{3}$ \\ ${ }^{1}$ Sixth Department of Radiation Oncology, Shandong Cancer Hospital; ${ }^{2}$ Shandong Academy of Medical Sciences; \\ ${ }^{3}$ Department of Radiotherapy Physics, Shandong Cancer Hospital, Jinan, P.R. China
}

Received March 10, 2011; Accepted June 23, 2011

DOI: 10.3892/etm.2011.301

\begin{abstract}
The aim of this study was to determine whether functional dose-volume histograms (FDVHs) are valuable for predicting radiation pneumonitis (RP), and to identify whether FDVHs have advantages over conventional dose-volume histograms (DVHs) for the prediction of RP in patients with locally advanced non-small cell lung cancer (LANSCLC). Fifty-seven patients with LANSCLC undergoing functional image-guided late-course accelerated hyperfractionated radiotherapy were enrolled. The grade of RP was evaluated according to the Common Toxicity Criteria 3.0. To identify predictive factors of RP, the FDVHs, including the volume of the functional lung receiving $5 \mathrm{~Gy}\left(\mathrm{FV}_{5}\right)$ through $50 \mathrm{~Gy}$ $\left(\mathrm{FV}_{50}\right)$, mean perfusion-weighted lung dose (MPWLD) and functional normal tissue complication probability (FNTCP), were analyzed and compared to their counterparts [total lung receiving 5 Gy $\left(\mathrm{V}_{5}\right)$ through 50 Gy $\left(\mathrm{V}_{50}\right)$, mean lung dose (MLD) and normal tissue complication probability (NTCP)] derived from conventional DVHs. Univariate analysis revealed that $\mathrm{V}_{5}-\mathrm{V}_{40}, \mathrm{MLD}$, NTCP and $\mathrm{FV}_{5}-\mathrm{FV}_{50}$, MPWLD, FNTCP were all statistically significant relative to the development of RP (all $\mathrm{p}<0.05$ ). Multivariate analysis showed that only MLD and $\mathrm{FV}_{15}$ were associated with RP ( $\mathrm{p}=0.001$ and 0.044 , respectively). Receiver operator characteristic curve anaysis
\end{abstract}

Correspondence to: Dr Baosheng Li, Sixth Department of Radiation Oncology, Shandong Cancer Hospital, Jiyan Road 440, Jinan, P.R. China

E-mail: baoshli@yahoo.com

Abbreviations: FDVHs, functional dose-volume histograms; MPWLD, mean perfusion-weighted lung dose; FNTCP, functional normal tissue compilation probability; RP, radiation pneumonitis; LANSCLC, locally advanced non-small cell lung cancer; FL, functional lung

Key words: non-small cell lung cancer, radiation pneumonitis, functional dose-volume histogram indicated that almost all of the FDVHs had larger areas under the curve compared to the DVHs, although no statistically significant difference was observed ( $p$-value ranged from 0.066 to 0.951 ). FDVHs are valuable for predicting RP with the predictive efficiency equivalent to or slightly advantageous over conventional DVHs. More homogeneous studies involving larger numbers of patients are required to further assess the value of FDVHs for predicting RP.

\section{Introduction}

Platinum-based chemoradiotherapy represents the current treatment standard for locally advanced non-small cell lung cancer (LANSCLC). However, treatment success is constrained by poor local control and radiation pneumonitis (RP). According to a systematic review (1), clinically significant RP usually develops in $13-37 \%$ of patients receiving radical dose radiation therapy for lung cancer. Despite the large number of studies $(2-8,23,24)$ involving clinical and dosimetric prognostic factors for RP, there are currently no validated and standardized factors for prediction. In clinical practice, the mean lung dose (MLD) and VDth (the volume of lung receiving more than a threshold dose) are the most common parameters used as predictors (2-7). However, these parameters are not ideal due to their limited accuracy, sensitivity and specificity (1). This may be explained by the fact that radiation ideally should be delivered in a manner that minimizes its functional consequences (9). For the most part, this goal has been sought by trying to minimize the volume of computed tomography-defined lung tissue within the treatment fields. This approach does not, however, consider possible variations in the functional competence of different regions of the lung. The same problem arises in the interpretation of dose-volume histograms (DVHs) and calculations of normal tissue complication probability (NTCP). From the viewpoint of biophysics, these parameters are constructed to consider both lungs as a homogeneous organ, however, conventional models do not take the possible spatial differences of lung radiosensitivity into account $(10,11,27)$. Furthermore, co-existent lung diseases in the majority of patients presenting with lung cancer result in regional differences in lung function. Nioutsikou et al (12) considered that the functional heteroge- 
neity of an organ as a factor contributes to the probability of complications in the normal tissues following radiotherapy. Using the functional dose-volume histograms (FDVHs) and the functional normal tissue complication probability (FNTCP) may be more meaningful for plan evaluation and is anticipated to show a better correlation with RP.

\section{Materials and methods}

Patient characteristics. Fifty-seven patients with stage III NSCLC enrolled in a prospective phase II study from March 2006 to April 2010 were analyzed. Eligibility criteria included biopsy-proven NSCLC, no prior chemotherapy or radiotherapy, no concurrent malignancy and no past history of lung cancer. The protocol was approved by the IRB, and informed consent was obtained from all patients. All patients received late-course accelerated hyperfractionated radiotherapy (LCAHRT) with three dimensional conformal (3D-CRT) or intensity-modulated radiotherapy (IMRT) techniques. The initial volume was treated with conventional fractionation to a total dose of 40 Gy in 2-Gy fractions over 4 weeks; the boost volume was irradiated with LCAHRT to additional doses of 19.6-39.2 Gy in 2 fractions of 1.4 Gy with an interval of 6-8 h per day, 5 days per week over 2-3 weeks. Fifty (88\%) patients received 2-4 cycles of concurrent or sequential chemotherapy with cisplatin-based regimens. Seventy-nine percent of patients had a history of smoking. The median baseline forced expiratory volume in $1 \mathrm{sec}\left(\mathrm{FEV}_{1.0}\right)$ was 2.08 liters (range 0.62-3.59). Patient characteristics are shown in Table I.

Treatment planning and delivery. All patients were scanned using a dedicated positron emission tomography/computed tomograpy (PET/CT; 4 slice Discovery LS; GE) simulator in the supine position with arms extended above the head and immobilized by a vacuum cradle device to improve the setup reproducibility during planning and delivery of treatment according to the ${ }^{18} \mathrm{~F}$-fluorodeoxyglucose $\left({ }^{18} \mathrm{~F}-\mathrm{FDG}\right) \mathrm{PET} / \mathrm{CT}$ imaging protocol (13).

Single photon emission computed tomography (SPECT; GE Infinia) scans were also performed in the treatment position on the next day, following injection of technetium-99m $\left({ }^{99 \mathrm{~m}} \mathrm{Tc}\right)$ labeled with macro-aggregated albumin (MAA) tracer. SPECT image acquisition and reconstruction were performed as previously described $(9,10,20)$. After the required SPECT lung perfusion imaging, these functional images were all transferred to a Philips Pinnacle ${ }^{3}$ planning system (Philips Radiation Oncology Systems, Milpitas, CA, USA), and then registered manually with the aid of fiducial markers $(12,14)$.

The ${ }^{18} \mathrm{~F}$-FDG PET/CT image was used to delineate the gross tumor volume (GTV), including the primary disease plus any involved regional lymph nodes as determined by size on the CT scan to be $\geq 1 \mathrm{~cm}$ or FDG-avid tumor and lymph nodes, regardless of their anatomic size. Before commencing the visual contouring, a diagnostically adequate window for image display was adjusted with the assistance of our nuclear medicine physician. The planning target volume (PTV) was considered to include the GTV plus a 10 - to $15-\mathrm{mm}$ margin. Ninety-five percent isodose line encompassed the PTV. Normal tissues (esophagus, spinal cord, heart and total lung) were contoured as usual. In particular, functional lung
Table I. Patient characteristics.

$\begin{array}{lr}\begin{array}{lr}\text { Characteristic } \\ \text { Gender }\end{array} & \begin{array}{r}\text { No. of patients } \\ \text { (ratio and \%) }\end{array} \\ \text { Male:female } & 48(84): 9(16) \\ \text { Age (years) } & 36(63): 21(37) \\ \geq 60:<60 & 28(49) \\ \text { Histopathology, n (\%) } & 20(35) \\ \text { Squamous cell carcinoma } & (5) \\ \text { Adenocarcinoma } & 6(11) \\ \text { Large cell carcinoma } & \\ \text { NSCLC, not otherwise specified } & 19(33): 38(67) \\ \text { Clinical stage } & \\ \text { IIIA:IIIB } & 54(95): 3(5) \\ \text { Karnofsky performance status } & \\ \geq 80:<80 & 12(21): 45(79) \\ \text { Smoking history } & \\ \text { No:yes } & 34(60): 16(28) \\ \text { Chemotherapy } & \\ \text { Concurrent:sequential } & \\ \end{array}$

Table II. Association of baseline characteristics with grade $\geq 2$ radiation pneumonitis (RP).

\begin{tabular}{lccc}
\hline Characteristic & $\begin{array}{c}\text { No-RP } \\
(\text { mean } \pm 1 \text { SD })\end{array}$ & RP & p-value $^{\mathrm{a}}$ \\
\hline FEV $_{1.0}$ (liters) & $2.2 \pm 0.7$ & $1.7 \pm 0.6$ & 0.06 \\
FVC (liters) $_{\text {FEV }_{1.0} / \text { FVC (\%) }}$ & $3.0 \pm 0.8$ & $2.5 \pm 0.9$ & 0.08 \\
\hline
\end{tabular}

$\mathrm{FEV}_{1.0}$, forced expiratory volume in $1 \mathrm{sec}$; FVC, forced vital capacity. ${ }^{a}$ Analyzed by independent-sample t-test.

(FL) was weighted by ${ }^{99 m}$ Tc-MAA SPECT lung perfusion. According to the study of Seppenwoolde et al (15), $\geq 30 \%$ of the maximum pre-RT perfusion was defined as 'well-perfused'. It is assumed that perfusion is proportional to function $(16,17)$. We delineated the regional well-perfused lung contours as FL. Based on the functional information, PET/CT/SPECT-guided radiotherapy planning was optimized $(14,18)$.

For 3D-CRT, four or five beams were consistently employed in the treatment plans, typically anterior-posterior beams in combination with oblique beams. In IMRT plans, five to seven beam angles were usually employed for dose optimization. Dose calculations were performed using Pinnacle $^{3}$ version 7.6c (ADAC, Milpitas, CA, USA) with tissue heterogeneity correction. Planning objective for organs at risk was defined as follows: total lung receiving $>20$ Gy $\left(\mathrm{V}_{20}\right)$ limited to $\leq 37 \%$; maximum dose of spinal cord limited to $\leq 45 \mathrm{~Gy}$; for heart, with constraints: $\mathrm{D}_{1 / 3} \leq 50 \mathrm{~Gy}$, $D_{2 / 3} \leq 45 \mathrm{~Gy}, \mathrm{D}_{3 / 3} \leq 40$ Gy $\left(\mathrm{D}_{\mathrm{x}}\right.$ is dose received by $\mathrm{x}$ of the volume). The treatment plans were reviewed by peers and 
delivered using $6 \mathrm{MV}$ beams on linear accelerators (21EX, 23EX or Trilogy; Varian Inc., CA, USA).

\section{Functional dose-volumetric parameters}

FDVHs and mean perfusion-weighted lung dose (MPWLD). After a SPECT scan was adequately registered with the PET/ CT dataset, the entire 3D-RT dose distribution was overlaid onto the SPECT scan. The traditional DVHs of the total lung volume defined by CT were converted into new histograms, which were termed FDVHs $(9,12,19,20)$. Based on these homogeneous functional sub-units, FDVHs were calculated from $\mathrm{FV}_{5}$ to $\mathrm{FV}_{50}$; meanwhile, conventional parameters $\mathrm{V}_{5}-\mathrm{V}_{50}$ were also calculated. The MLD was defined as the average dose throughout the total lung volume minus the GTV. Subsequently, the MLD was weighted with the local perfusion resulting in the MPWLD $(10,20,26)$. The adjustment of the dose to the biologically equivalent dose by conventional fractionation size at 2 Gy was not carried out.

Functional normal tissue complication probability. The Lyman model was used to calculate the NTCP from the DVHs. Assumptions used in the calculation were: $\mathrm{n}=0.87 ; \mathrm{m}=0.18$; $\mathrm{TD}_{50}=24.5 \mathrm{~Gy}$ (21). The FNTCP was calculated from the FDVHs with the same $n, m$ and $\mathrm{TD}_{50}$ values, and was used as an additional parameter.

Evaluation of $R P$ and follow-up. After completion of treatment, patients were followed up at 3-month intervals until RP was observed or the patients died. In our study, follow-up time ranged from 3 to 42 months, with follow-up for living patients determined to be a median of 14 months. In our analysis, the RP grade was defined according to the National Cancer Institute Common Toxicity Criteria, version 3.0 (22). The development of RP was considered as a binary variable: no-RP (RP grade $\leq 1)$ and RP (RP grade $\geq 2$ ).

Statistical analysis. The Chi-square test was applied to test patient characteristic distribution between the no-RP and RP groups. Univariate and multivariate analyses were used to evaluate the impact of DVHs and FDVHs on the development of RP. For the multivariate analysis, backward stepwise logistic regression analysis was adopted. Receiver operator characteristic (ROC) curves were used to identify the reference threshold of FDVHs and to assess the predictability of the parameters. The correlation between the FDVHs and DVHs was tested by calculating the Pearson correlation coefficient. All statistical tests were two-tailed and were performed using statistical software programs Medcalc V.11.2 and SPSS V.11.5. A p-value of $<0.05$ was considered significant.

\section{Results}

Clinical parameters. In the present study, $36(63 \%)$ patients did not develop RP, 10 (18\%) patients developed grade 1, 7 (12\%) grade 2, $3(5 \%)$ grade 3 and 1 grade $5 \mathrm{RP}$. The actuarial development of grade 2 or worse RP was $19.3 \%$. There was no significant difference in the distribution of clinical parameters (gender, age, Karnofsky performance status, smoking history and chemotherapy) between the two groups of patients (no-RP vs. RP). FEV 1.0 and forced vital capacity (FVC) investigated at borderline exhibited significance $(\mathrm{p}=0.06$ and 0.08 , respectively), where $\mathrm{FEV}_{1.0} / \mathrm{FVC}$ did not $(\mathrm{p}=0.90)$ (Table II).

Dose-volumetric parameters. Tables III and IV summarize the analysis of the potential predictors of DVHs to RP. $V_{5}-V_{40}$, MLD and NTCP were statistically significant, whereas multivariate analysis showed that MLD was the only risk factor $(\mathrm{p}=0.001)$.

Tables III and V summarize the analysis of the potential predictors of FDVHs to RP. FV $-\mathrm{FV}_{50}$, MPWLD and FNTCP were all statistically significant, whereas multivariate logistic regression analysis showed that $\mathrm{FV}_{15}$ was the most important risk factor $(\mathrm{p}=0.044)$.

Table VI reports the sensitivity and specificity of FDVHs for RP prediction. Using ROC curve analysis, all of the FDVHs, with the exception of MPWLD, had larger AUCs than their counterparts from DVHs, although no significant difference

Table III. Univariate analysis of the association of FDVHs (DVHs) with grade $\geq 2$ radiation pneumonitis (RP).

\begin{tabular}{|c|c|c|c|}
\hline Parameter & Threshold & RP rate $(\%)$ & $\mathrm{p}$-value \\
\hline $\mathrm{FV}_{5}\left(\mathrm{~V}_{5}\right)$ & $0.8022(0.7073)$ & $5.3^{\mathrm{b}}: 47.4^{\mathrm{c}}(5.4: 45.0)$ & 0.001 \\
\hline $\mathrm{FV}_{10}\left(\mathrm{~V}_{10}\right)$ & $0.4538(0.5603)$ & $5.4: 45.0(5.1: 50.0)$ & 0.001 \\
\hline $\mathrm{FV}_{15}\left(\mathrm{~V}_{15}\right)$ & $0.3933(0.4069)$ & $2.9: 43.5(0.0: 39.3)$ & 0.001 \\
\hline $\mathrm{FV}_{20}\left(\mathrm{~V}_{20}\right)$ & $0.2961(0.3218)$ & $5.6: 42.9(3.2: 38.5)$ & 0.001 \\
\hline $\mathrm{FV}_{25}\left(\mathrm{~V}_{25}\right)$ & $0.2778(0.2606)$ & $9.1: 53.8(5.9: 39.1)$ & $0.001(0.004)$ \\
\hline $\mathrm{FV}_{30}\left(\mathrm{~V}_{30}\right)$ & $0.2086(0.2124)$ & $7.7: 44.4(8.6: 36.4)$ & $0.002(0.015)$ \\
\hline $\mathrm{FV}_{35}\left(\mathrm{~V}_{35}\right)$ & $0.1518(0.1686)$ & 7.3:50.0 (7.9:42.1) & $0.001(0.004)$ \\
\hline $\mathrm{FV}_{40}\left(\mathrm{~V}_{40}\right)$ & $0.1412(0.1669)$ & $7.1: 53.3(7.1: 31.0)$ & $0.001(0.041)$ \\
\hline $\mathrm{FV}_{45}\left(\mathrm{~V}_{45}\right)$ & $0.1052(0.1330)$ & $7.1: 53.3(9.7: 30.8)$ & $0.001(0.089)$ \\
\hline $\mathrm{FV}_{50}\left(\mathrm{~V}_{50}\right)$ & $0.0721(0.1083)$ & $7.1: 53.3(12.8: 37.5)$ & $0.001(0.061)$ \\
\hline MPWLD (MLD) (Gy) & $17.051(18.734)$ & $5.5: 42.9(2.4: 66.7)$ & 0.001 \\
\hline FNTCP (NTCP) & $0.0105(0.0742)$ & $0.0: 40.7(8.3: 77.8)$ & 0.001 \\
\hline
\end{tabular}

MPWLD, mean perfusion-weighted lung dose; MLD, mean lung dose; FNTCP, functional normal tissue complication probability. ${ }^{\mathrm{b}} \mathrm{RP}$ rate calculated when $\mathrm{V}_{\mathrm{x}}<$ threshold, ${ }^{\mathrm{c}} \mathrm{RP}$ rate calculated when $\mathrm{V}_{\mathrm{x}} \geq$ threshold. 
Table IV. Multivariate analysis of the association of DVHs with grade $\geq 2$ radiation pneumonitis.

\begin{tabular}{lcccc}
\hline Parameter & B & SE & Ward & p-value \\
\hline MLD & 4.422 & 1.178 & 14.086 & 0.001 \\
\hline
\end{tabular}

MLD, mean lung dose.
Table V. Multivariate analysis of the association of FDVHs with grade $\geq 2$ radiation pneumonitis.

\begin{tabular}{lcccc}
\hline Parameters & B & SE & Ward & p-value \\
\hline $\mathrm{FV}_{5}$ & 2.436 & 1.335 & 3.332 & 0.068 \\
$\mathrm{FV}_{15}$ & 2.815 & 1.398 & 4.056 & 0.044 \\
\hline
\end{tabular}

Table VI. ROC curve analysis for the association of FDVHs with grade $\geq 2$ radiation pneumonitis.

\begin{tabular}{|c|c|c|c|c|c|}
\hline Parameter & Threshold & Sensitivity (95\% CI) & Specificity (95\% CI) & AUC & $\mathrm{p}$-value \\
\hline $\mathrm{FV}_{5}$ & 0.8022 & $0.8182(0.482-0.977)$ & $0.7826(0.636-0.891)$ & 0.833 & 0.0001 \\
\hline $\mathrm{FV}_{10}$ & 0.4538 & $0.8182(0.482-0.972)$ & $0.7826(0.636-0.890)$ & 0.844 & 0.0001 \\
\hline $\mathrm{FV}_{15}$ & 0.3933 & $0.9091(0.587-0.998)$ & $0.7391(0.589-0.857)$ & 0.869 & 0.0001 \\
\hline $\mathrm{FV}_{20}$ & 0.2961 & $0.8182(0.482-0.972)$ & $0.7391(0.589-0.857)$ & 0.844 & 0.0001 \\
\hline $\mathrm{FV}_{25}$ & 0.2778 & $0.6364(0.309-0.888)$ & $0.8913(0.764-0.963)$ & 0.828 & 0.0001 \\
\hline $\mathrm{FV}_{30}$ & 0.2086 & $0.7273(0.391-0.937)$ & $0.8043(0.661-0.906)$ & 0.817 & 0.0001 \\
\hline $\mathrm{FV}_{35}$ & 0.1518 & $0.7273(0.391-0.937)$ & $0.8261(0.686-0.922)$ & 0.798 & 0.0001 \\
\hline $\mathrm{FV}_{40}$ & 0.1412 & $0.7273(0.391-0.937)$ & $0.8696(0.737-0.950)$ & 0.798 & 0.0002 \\
\hline $\mathrm{FV}_{45}$ & 0.1052 & $0.7273(0.391-0.937)$ & $0.7478(0.711-0.936)$ & 0.789 & 0.0003 \\
\hline $\mathrm{FV}_{50}$ & 0.0721 & $0.7273(0.390-0.940)$ & $0.8478(0.711-0.937)$ & 0.760 & 0.0008 \\
\hline MPWLD (Gy) & 17.051 & $0.8333(0.516-0.979)$ & $0.7556(0.605-0.857)$ & 0.844 & 0.0001 \\
\hline FNTCP & 0.0105 & $1.0000(0.735-1.000)$ & $0.6667(0.510-0.810)$ & 0.861 & 0.0001 \\
\hline
\end{tabular}

ROC, receiver operator characteristics; CI, confidence interval; AUC, area under the ROC curve; MPWLD, mean perfusion-weighted lung dose; FNTCP, functional normal tissue complication probability.

A

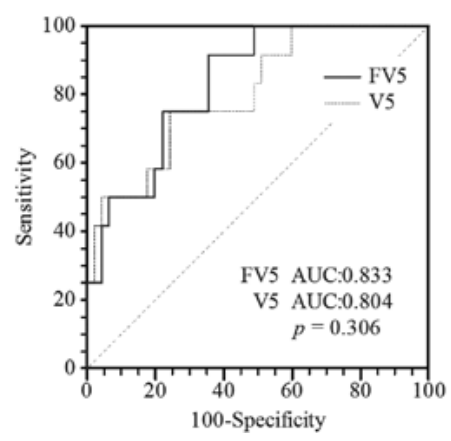

$\mathbf{E}$

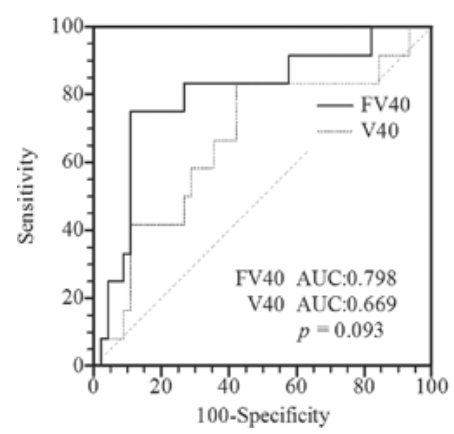

B

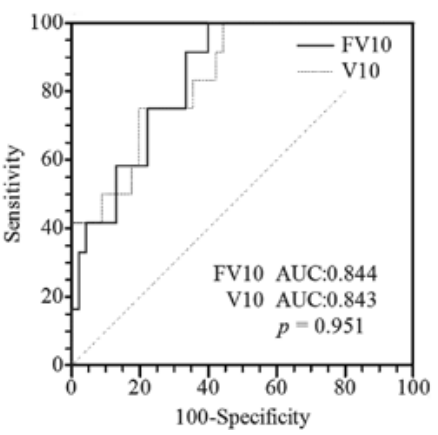

$\mathbf{F}$

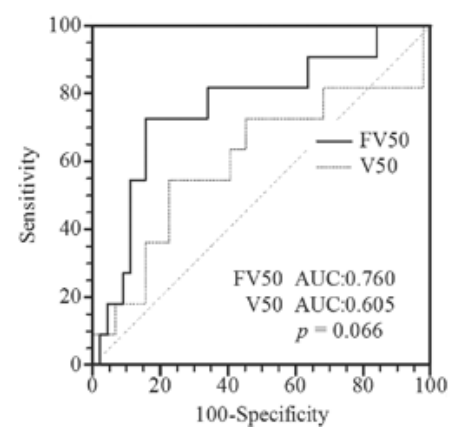

C

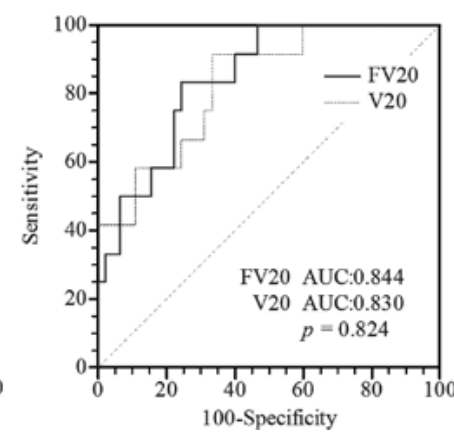

G

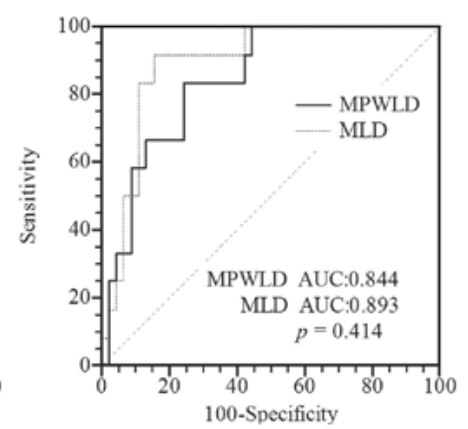

D

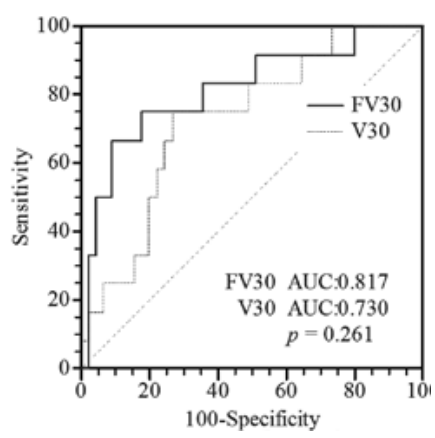

$\mathbf{H}$

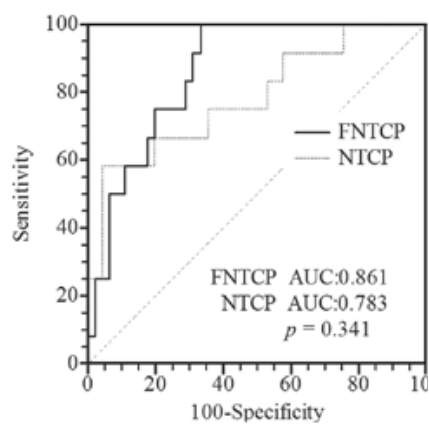

Figure 1. ROC curve comparison for predictive value between FDVHs and DVHs. AUC, areas under the curve. (A) $\mathrm{FV}_{5}$ vs. $\mathrm{V}_{5}$, (B) $\mathrm{FV}_{10}$ vs. $\mathrm{V}_{10}$, (C) $\mathrm{FV} V_{20}$ vs. $\mathrm{V}_{20}$, (D) $\mathrm{FV}_{30}$ vs. $\mathrm{V}_{30}$, (E) $\mathrm{FV}_{40}$ vs. $\mathrm{V}_{40}$, (F) $\mathrm{FV}_{50}$ vs. $\mathrm{V}_{50}$, (G) MPWLD vs. MLD and (H) FNTCP vs. NTCP. 
was observed (Fig. 1). Close correlations between FDVHs and DVHs were found (range of Spearman r, 0.725-0.940; $<<0.001$ ).

\section{Discussion}

The present results indicate that multiple conventional and functional dosimetric parameters can be predictors of RP for patients with LANSCLC, who were treated with LCAHRT. The predictive efficiency using functional parameters tend to be slightly improved compared to conventional counterparts.

Currently, although numerous studies have investigated predictive factors for RP, there are no validated and standardized methods for prediction. In particular, dosimetric factors have mostly been reported using conventionally fractionated radiotherapy schedules, and their reliability while using LCAHRT is unknown. Jenkins et al (23) reported that $\mathrm{V}_{20}$ and MLD were useful factors for predicting the risk of RP after treatment with continuous hyperfractionated accelerated radiotherapy (CHART). In terms of our study, many dose-volumetric parameters $\left(\mathrm{V}_{5}-\mathrm{V}_{40}\right.$, MLD and NTCP) were significantly correlated with an increased risk of RP. Among these parameters, MLD was shown to be the only independent predictor by multivariate analysis. A previous study (24) using different theoretical models generated a different single parameter from the DVHs, for example, MLD and VDth, to estimate the predictive value, which confirmed that MLD was the most accurate predictor for the incidence of RP, although the correlation between VDth and MLD was strong.

Compared to conventional DVHs, FDVHs were correlated with the local FL injury. In previous studies $(9,15,16,19,25,26)$, regional lung damage, assessed by SPECT perfusion combining the 3D dose distribution of individual patients with the average dose-effect relations, was predictive for the radiation-induced change in the overall pulmonary function, and possibly for the incidence of RP. Seppenwoolde et al (10) reported a statistically significant correlation between the incidence of RP and the MPWLD. In our initial study, $\mathrm{FV}_{5}-\mathrm{FV}_{50}$, MPWLD and FNTCP were all strongly correlated with RP, while multivariate analysis showed that only $\mathrm{FV}_{15}$ remains significant. For the promise of functional parameters, we evaluated their performance using ROC curve analysis compared to conventional equivalents. Unfortunately, the results of this study were quite unexpected, with MPWLD exhibiting a slight reduction in predictive efficiency compared to MLD (Fig. 1). Although almost all of the FDVHs had larger AUC than DVHs, the predictive efficiency of FDVHs among $\mathrm{FV}_{5}-\mathrm{FV}_{40}$ did not increase significantly, with the exception of $\mathrm{FV}_{50}$ with sensitivity $72.73 \%$ (95\% CI $0.39-0.94$ ), specificity $84.74 \%$ (95\% CI $0.71-0.94$ ) and accuracy $76.0 \%$ (area under the ROC curve) which was of borderline significance to $\mathrm{V}_{50}$. Kocak et al (20) prospectively tested the predictive abilities of dosimetric/functional parameters on two cohorts of patients from the Duke and Netherlands Cancer Institute (NKI). This previous study reported that perfusion weighted parameters $\left(\mathrm{FV}_{20}, \mathrm{FV}_{25}\right.$ and $\left.\mathrm{FV}_{30}\right)$ had equivalent or slightly larger ROC areas ( 0.55 vs. $0.54,0.54$ vs. 0.52 and 0.54 vs. 0.51 , respectively) than using conventional counterparts in the Duke cohort. In the NKI data, MPWLD appeared to be the slightly advantaged predictor over MLD (0.71 vs. 0.61). From these studies, we considered that FDVHs are valuable for predicting RP with the predictive efficiency no worse at least than conventional DVHs. The results of our study further revealed that the FDVHs had a strong relationship with DVHs.

Contemplating the present study, several factors may confine the predictive outcome of FDVHs. Firstly, in our study, we considered a perfusion value of $30 \%$ or more of the maximum radioactivity to be functional and the remaining regional lung was not (15). In fact, normal lung function is similar to a spectrum; the use of a $30 \%$ cutoff point creates FL and non-FL, which may result in losing parts of the 'non-FL' information or underestimating the function in these regions. Secondly, adequate lung function requires both ventilation and perfusion. Furthermore, clinical studies $(3,4,8)$ have found that the incidence of pneumonitis is higher in patients with lower lobe tumors. Therefore, further study combining anatomical information with ventilation/perfusion (V/Q) scan weighted functional dose-volumetric parameters should be investigated. Finally, we compared the efficiency between FNTCP and NTCP only as an experiment. If $\mathrm{m}, \mathrm{n}$ and $\mathrm{TD}_{50}$ were unchanged in the Lyman model, the area under the ROC curve of FNTCP was larger than NTCP, although no statistical significance was observed. Ideally, FNTCP should be calculated using FL parameters, which would be more meaningful (12). Further study involving functional $\mathrm{m}(\mathrm{mf}), \mathrm{n}(\mathrm{nf}), \mathrm{TD}_{50}\left(\mathrm{TD}_{50 \mathrm{f}}\right)$ and FNTCP calculated based on these functional parameters should be established, which may be a superior predictor to their counterpart.

In summary, this study suggests that multiple conventional DVHs and FDVHs may be predictors of RP for LANSCLC patients treated with LCAHRT. The predictive efficiency of FDVHs is equivalent to or slightly advantageous over DVHs. More homogeneous studies involving larger numbers of patients are required to further assess the value of FDVHs in the prediction of RP.

\section{Acknowledgements}

This study was supported, in part, by the National Nature Science Foundation (grant no. 30970861) and by the Science and Technology Project of Shandong Province (2006GG2202012 and 2009GG10002011).

\section{References}

1. Rodrigues G, Lock M, D'Souza D, et al: Prediction of radiation pneumonitis by dose-volume histogram parameters in lung cancer - a systematic review. Radiother Oncol 71: 127-138, 2004.

2. Fay M, Tan A, Fisher R, et al: Dose-volume histogram analysis as predictor of radiation pneumonitis in primary lung cancer patients treated with radiotherapy. Int $\mathrm{J}$ Radiat Oncol Biol Phys 61: 1355-1363, 2005.

3. Claude L, Pérol D, Ginestet C, et al: A prospective study on radiation pneumonitis following conformal radiation therapy in non-small cell lung cancer: clinical and dosimetric factors analysis. Radiother Oncol 71: 175-181, 2004.

4. Graham MV, Purdy JA, Emami B, et al: Clinical dose-volume histogram analysis for pneumonitis after 3D treatment for non-small cell lung cancer (NSCLC). Int J Radiat Oncol Biol Phys 45: 323-329, 1999.

5. Kim TH, Cho KH,Pyo HR, et al: Dose-volumetric parameters for predicting severe radiation pneumonitis after three-dimensional conformal radiation therapy for lung cancer. Radiology 235: 208-215, 2005.

6. Oh D, Ahn YC, Park HC, et al: Prediction of radiation pneumonitis following high-dose thoracic radiation therapy by $3 \mathrm{~Gy} /$ fraction for non-small cell lung cancer: analysis of clinical and dosimetric factors. Jpn J Clin Oncol 39: 151-157, 2009. 
7. Piotrowski T, Matecka-Nowak M and Milecki P: Prediction of radiation pneumonitis: dose-volume histogram analysis in 62 patients with non-small cell lung cancer after three-dimensional conformal radiotherapy. Neoplasma 52: 56-62, 2005.

8. Yorke ED, Jackson A, Rosenzweig KE, et al: Dose-volume factors contributing to the incidence of radiation pneumonitis in nonsmall-cell lung cancer patients treated with three-dimensional conformal radiation therapy. Int J Radiat Oncol Biol Phys 54: 329-339, 2002.

9. Marks LB, Spencer DP, Sherouse GW, et al: The role of three dimensional functional lung imaging in radiation treatment planning: the functional dose-volume histogram. Int J Radiat Oncol Biol Phys 33: 65-75, 1995.

10. Seppenwoolde Y, de Jaeger K, Boersma LJ, et al: Regional differences in lung radiosensitivity after radiotherapy for nonsmall-cell lung cancer. Int J Radiat Oncol Biol Phys 60: 748-758, 2004.

11. Marks LB and Prosnitz LR: Estimation of normal tissue complication probabilities with three-dimensional technology. Int J Radiat Oncol Biol Phys 28: 777-779, 1994.

12. Nioutsikou E, Partridge M, Bedford JL, et al: Prediction of radiation-induced normal tissue complications in radiotherapy using functional image data. Phys Med Biol 50: 1035-1046, 2005.

13. Nestle U, Kremp S and Grosu AL: Practical integration of (18F)-FDG-PET and PET-CT in the planning of radiotherapy for non-small cell lung cancer (NSCLC): the technical basis, ICRUtarget volumes, problems, perspectives. Radiother Oncol 81: 209-225, 2006

14. Lavrenkov K, Christian JA, Partridge M, et al: A potential to reduce pulmonary toxicity: the use of perfusion SPECT with IMRT for functional lung avoidance in radiotherapy of non-small cell lung cancer. Radiother Oncol 83: 156-162, 2007.

15. Seppenwoolde Y, Muller SH, Theuws JC, et al: Radiation doseeffect relations and local recovery in perfusion for patients with non-small cell lung cancer. Int J Radiat Oncol Biol Phys 47: 681-690, 2000

16. Boersma LJ, Damen EM, de Boer RW, et al: A new method to determine dose-effect relations for local lung-function changes using correlated SPECT and CT data. Radiother Oncol 29: 110-116, 1993.
17. Fan M, Marks LB, Hollis D, et al: Can we predict radiationinduced changes in pulmonary function based on the sum of predicted regional dysfunction? J Clin Oncol 19: 543-550, 2001.

18. Seppenwoolde Y, Engelsman M, de Jaeger K, et al: Optimizing radiation treatment plans for lung cancer using lung perfusion information. Radiother Oncol 63: 165-177, 2002.

19. Marks LB, Munley MT, Spencer DP, et al: Quantification of radiation-induced regional lung injury with perfusion imaging. Int J Radiat Oncol Biol Phys 38: 399-409, 1997.

20. Kocak Z, Borst GR, Zeng J, et al: Prospective assessment of dosimetric/physiologic-based models for predicting radiation pneumonitis. Int J Radiat Oncol Biol Phys 67: 178-186, 2007.

21. Emami B, Lyman J, Brown A, et al: Tolerance of normal tissue to therapeutic irradiation. Int J Radiat Oncol Biol Phys 21: 109-122, 1991.

22. Trotti A, Colevas AD, Setser A, et al: CTCAE v3.0: development of a comprehensive grading system for the adverse effects of cancer treatment. Semin Radiat Oncol 13: 176-181, 2003.

23. Jenkins P, D'Amico K, Benstead K, et al: Radiation pneumonitis following treatment of non-small cell lung cancer with continuous hyperfractionated accelerated radiotherapy (CHART). Int J Radiat Oncol Biol Phys 56: 360-366, 2003.

24. Seppenwoolde Y, Lebesque JV, de Jaeger K, et al: Comparing different NTCP models that predict the incidence of radiation pneumonitis. Normal tissue complication probability. Int J Radiat Oncol Biol Phys 55: 724-735, 2003.

25. Boersma LJ, Damen EM, de Boer RW, et al: Estimation of overall pulmonary function after irradiation using dose-effect relations for local functional injury. Radiother Oncol 36: 15-23, 1995.

26. De Jaeger K, Seppenwoolde Y, Boersma LJ, et al: Pulmonary function following high-dose radiotherapy of non-small cell lung cancer. Int J Radiat Oncol Biol Phys 55: 1331-1340, 2003.

27. Liao ZX, Travis EL and Tucker SL: Damage and morbidity from pneumonitis after irradiation of partial volumes of mouse lung. Int J Radiat Oncol Biol Phys 32: 1359-1370, 1995. 